\title{
Assessing the Outcome of Management of Thyroid Dysfunction in Pregnancy With Second Affiliated Hospital of Chongqing Medical University Guideline.
}

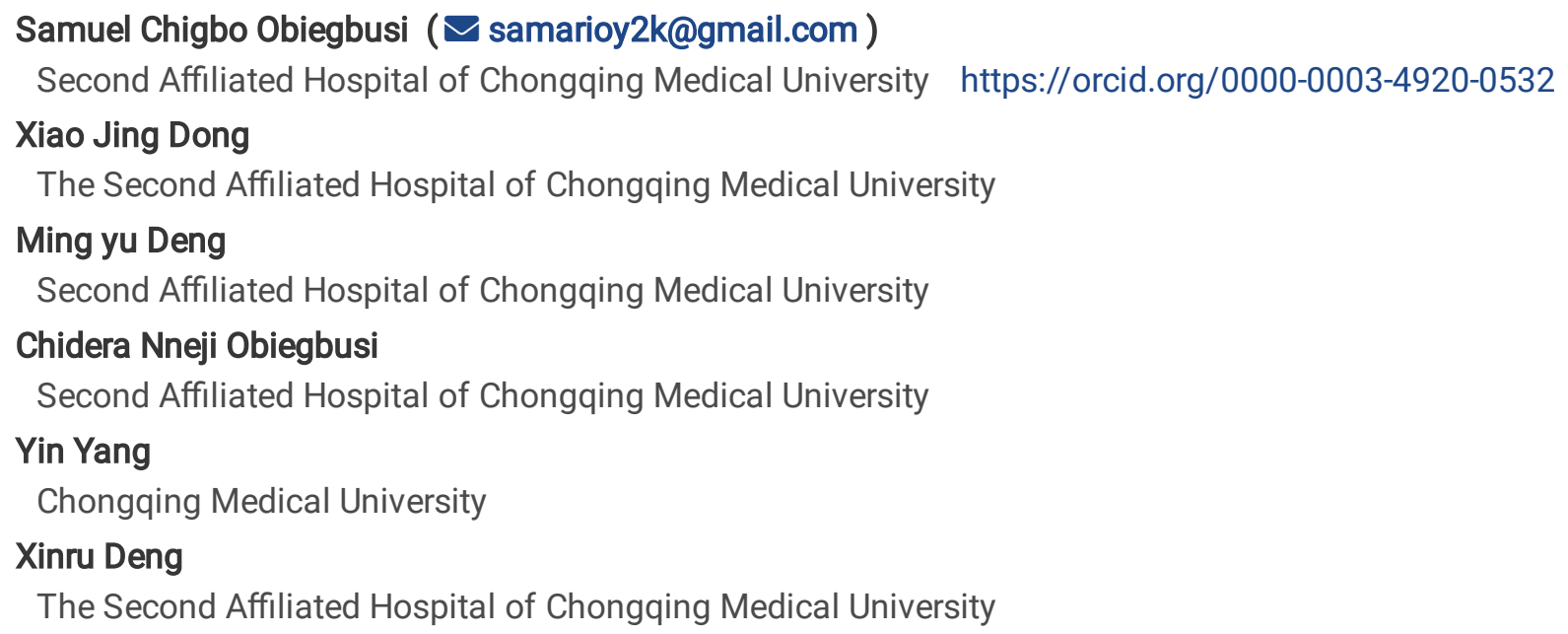

\section{Research Article}

Keywords: Pregnancy, Thyroid dysfunction, Thyroid screening, Hypothyroidism, subclinical hypothyroidism, complication, guideline

Posted Date: March 8th, 2021

DOI: https://doi.org/10.21203/rs.3.rs-252844/v1

License: (c) (i) This work is licensed under a Creative Commons Attribution 4.0 International License. Read Full License 


\section{Abstract}

Introduction - Pregnancy comes with hormonal changes which, when not properly managed, could lead to complications. Thyroid hormone is one of the hormones that are affected during pregnancy, and it plays a significant role in pregnancy, from conception to delivery. In a bid to identify intended pregnant women and pregnant women with thyroid dysfunction, the Endocrinology Branch of Chinese Medical Association and Perinatal medicine branch of Chinese Medical Association set guidelines for diagnosis and treatment of thyroid diseases in pregnancy and postpartum women. The guideline recommends screening for all women who desire getting pregnant soon and pregnant women, which Second Affiliated Hospital of Chongqing Medical University is implementing.

Purpose - To Identify the common thyroid disease found among pregnant women in Chongqing. Evaluate the effectiveness of the management guideline toward improving pregnancy outcome among women diagnosed with thyroid disease during their pregestational and gestational period, and ascertain the need for additional measures to be taken towards thyroid disease management during pregnancy in certain areas with unfavourable outcome.

Method - A retrospective cohort study of 774 pregnant women diagnosed with thyroid dysfunction in the Second Affiliated Hospital of Chongqing Medical University from 2016 -2018 was extracted from the hospital computer patient's record. Only 724 patients that met the inclusive criteria were analysed. Participants were grouped into four, according to the time they were diagnosed and managed. The Multiple logistic regression and binary logistic regression statistical analysis were done with SPSS, and we adjusted for potential confounders, including maternal age, parity, and gravida.

Result - There is an association between maternal age and abortion among pregnant women diagnosed with subclinical hypothyroidism, $P-0.018(O R$ 1.459, 95\% Cl 1.067-1.997) and significant difference in pregnant women who developed intrauterine growth restriction after being diagnosed with hypothyroidism in the second trimester, $P-0.048(0 R-0.152,95 \% \mathrm{Cl}$ $0.024-0.981)$. There was also a significant difference in gravida, $P-0.032(O R 1.368,95 \% \mathrm{Cl} 1.0281 .821)$ and normal delivery mode, $P-0.010$ (OR 2.521, 95\% Cl 1.246-5.100).

Conclusion - The study shows a promising result as less complication is observed. However, more attention is needed toward managing subclinical hypothyroidism in pregnancy to curb abortion/miscarriage incidence. Hypothyroidism in second trimester could lead to intrauterine growth restriction. Multigravida increases the risk of complication among pregnant women with thyroid dysfunction.

\section{Introduction}

Several investigations report that at least 2 to $3 \%$ of healthy, non-pregnant women of childbearing age have an elevated serum thyroid-stimulating hormone (TSH). The prevalence may be higher in areas of iodine insufficiency [1]. Thyroid disorders are the second most common endocrine disorders found in pregnancy. Overt hypothyroidism is estimated to occur in $0.3-0.5 \%$ of pregnancies. Subclinical hypothyroidism appears to occur in $2-3 \%$, and hyperthyroidism is present in $0.1-0.4 \%$ [2]. The study on thyroid hormone and its role during pregnancy and childbearing has been a major concern today. Limited research and evidence in this vital area have prompt lots of research in this field in recent times. These thyroid disorders include clinical hypothyroidism, Subclinical hypothyroidism, hypothyroxinemia, hyperthyroidism, thyroiditis, and other thyroid dysfunction.

The primary cause of Hypothyroidism or subclinical hypothyroidism (SCH) in developing countries is iodine deficiency, while in developed countries it is caused by chronic autoimmune thyroiditis (CAT). Thyroid autoantibodies are detected in about $50 \%$ of pregnant women with subclinical hypothyroidism and more than $80 \%$ with overt hypothyroidism [3]. Hence, both developed and developing nations are in one way or the other at risk of developing thyroid disease or experiencing thyroid dysfunction. Increased and decreased thyroid hormone levels result from various factors like autoimmune condition, tumour, infection, metabolic disorder, inflammation, and low feedback mechanism. It could also be due to physiological hormonal changes that occur during pregnancy. CAT is the leading cause of hypothyroidism during pregnancy [4]. Normal pregnancy is accompanied by a physiologic rise in thyroid hormone synthesis, facilitated by an increase in thyroid size by $10 \%$ in iodide 
sufficient areas and 20 to $40 \%$ in iodide deficient regions [1]. During pregnancy, the thyroid hormone needed to maintain the mother and foetus's metabolic activities depends mostly upon the mother's thyroid hormone release, especially in the first trimester, and gradually reduces as the pregnancy advances due to thyroid hormone production by the foetus [5]. This gradual reduction in demand on the maternal thyroid hormone specifically T4 is due to the fetus's ability to produce its thyroid hormone by the eleventh week of gestation, as demanded by the body [6].

However, it is a fact that the thyroid hormone plays a significant role in placenta formation. Hence, it can determine the outcome of pregnancy on both the mother and foetus. Similarity has been found between hyperthyroidism and hypothyroidism complications during prenatal, natal, and postnatal phases between the mother and baby. Among these similar complications found on the maternal side are; preterm delivery, preeclampsia, miscarriage, placenta abruption, and cardiac dysfunction [7]. While on the foetal/neonatal side are prematurity, low birth weight, congenital anomalies, and stillbirth [7]. These unpleasant outcomes have made thyroid hormone management a thing of importance to improve maternal and foetal wellbeing during pregnancy. Failure to manage these conditions will likely amount to complications, and undesirable outcomes, as seen in Parizad et al. [8] where preterm birth incidence was higher among mothers with clinical hypothyroidism, subclinical hypothyroidism, and hypothyroxinemia during pregnancy compared to euthyroid mothers, with a p-value of 0.005 , 0.013 , and 0.020 , respectively.

Despite the significant impact of thyroid hormone abnormality in pregnancy, it is still a thing of deliberation on whether to conduct a routine screening test for all pregnant women or not. The following may be the reason for this indecisive approach towards this critical health condition that needs a proactive and intense management policy. Firstly, insufficient statistical evidence of the true prevalence of this condition worldwide, as studies carried out in India and some middle-east nations have shown that subclinical hypothyroidism has a prevalence rate of $13.13 \%$ [9], against the western world with a prevalence rate of $3 \%$ [10]. Secondly, the cost of screening may have been perceived as a burden to the patients or the government in countries offering free health care to her masses. Thirdly, to date, there is no standard or uniform upper and lower limit (value) for the diagnosis of hypo and hyperthyroidism. Various nations, provinces, states, districts, and even hospitals have their range of value for diagnosing this condition, hence making it more challenging to initiate screening since some studies have shown a significant discrepancy in thyroid hormone value during pregnancy across races. Considering the influence of race and its physiologic variation, it becomes a thing of great importance to identify what works for specific population, community, nation, and race, rather than emulating that which works for others. Two studies from China and one study from India, for example, demonstrated a significantly higher TSH reference range for each trimester; in particular, the study by Li et al.[11], showed that the Chinese population displays $0.12-5.08 \mathrm{mU} / \mathrm{I}$ as first-trimester reference range; as a consequence, using the suggested $0.1-2.5 \mathrm{mU} / \mathrm{I}$ as reference range means that about $28 \%$ of the pregnant women in China would suffer from hypothyroidism, versus $4 \%$ when using an ethnically specific reference range [10].

Due to the variation in thyroid hormone value across geographical location, one of the recommendations in 2017 American Thyroid Association (ATA) guideline in management of thyroid disease during pregnancy is that pregnancy-specific population-based reference intervals should be determined locally. However, if the TSH trimester-specific reference ranges are not available in the laboratory, the following reference range upper limits are recommended: First trimester $0.1-2.5 \mathrm{mU} / \mathrm{l}$; second trimester, $0.2-3.0 \mathrm{mU} / \mathrm{l}$; third trimester, 0.3-3.5mU/I [12]. A recent guideline recommends that, when available, the population and trimester-specific reference ranges for serum TSH during pregnancy should be defined by a provider's institute or laboratory and should represent the specific population for whom care is provided. If those are not available, an upper reference limit of near $4.0 \mathrm{mU} / \mathrm{L}$ may be used $[1,12]$. Most endocrinologists were convinced about an evident association between mild thyroid impairment and adverse outcomes in pregnancy, thus using a TSH value of $2.5 \mathrm{mU} / \mathrm{L}$ as the threshold for diagnosing hypothyroidism and starting levothyroxine in pregnant women [13].

In China, the issue of standard cut-off in upper and lower value of thyroid hormone in pregnant women is not different. These values vary in various hospitals, depending on the gestational age. This reference range variation could be identified in table 1 and 2 below, between Chongqing and Nanjing hospital reference range. 
According to the Compilation by the Committee of "Guidelines for Diagnosis and Treatment of Thyroid Diseases in Pregnancy and postpartum [15], reference range should be generated and set by various region/health institution/community as recommended below:

1. To diagnose thyroid dysfunction during pregnancy, there is need to establish a method to ascertain specific reference range at each gestational period (early, middle and late) for serum thyroid function index (TSH, FT4, TT4).

2. The method recommended by National Academia of Clinical Biochemistry (NACB) to develop a reference range by choosing areas with adequate iodine, single births, no previous thyroid disease, and thyroid gland disease. For pregnant women with negative body antibodies and no goitre, the reference range is $2.5 \sim 97.5$ percentile.

Because various health care institutions have different methods and means of testing thyroid function, it is more challenging to have a harmonised reference range. The variation in the test reagents and kits was addressed by providing a pregnancyspecific TSH and Free thyroxine(FT4) trimester reference range used in hospitals that use the same kit [16-21], as seen in table 3.

Identification of pregnant women with thyroid dysfunction is essential, but without proper management, the aim of screening will be defeated. The discrepancy in diagnostic reference range will lead to variation in management approach, knowing that this disease's physiologic effect varies in various society, community, and group of people. Hence, there is a need to establish a management guideline that meets the target population's aims and goals.

In China, this management guideline was set by the endocrinology branch of the Chinese Medical Association and Perinatal medicine branch of the Chinese Medical Association to meet the expected management goals. The guideline for the management of patients in this category is shown in table 4 .

Prevention of possible complications associated with thyroid hormone disorder and other pregnancy abnormalities should be the utmost priority for Obstetricians and Gynaecologists. This can be achieved by screening all pregnant women regardless of their perceived threat for thyroid dysfunction or not. Unfortunately, universal screening of asymptomatic pregnant women for thyroid disease during their antenatal visit without possible indication(s) is still controversial. Due to insufficient evidence and unsatisfactory criteria for universal screening, these patients who could be diagnosed and managed earlier are left at the doctor's discretion. Most times, the doctors fail to identify these patients due to limited medical and family history. As well, these patients might present without any clinical signs and symptoms that will suggest possible thyroid screening. These missed women with thyroid dysfunction could be due to similarity in their clinical presentation of thyroid dysfunction and pregnancy physiology, such as constipation, weight gain and fatigue. According to research conducted in the Middle East, $27 \%$ of pregnant women with thyroid disease were undiagnosed by the doctors. This is also shown in the study conducted by Vaidya et al. [22] which demonstrated that focusing screening on only suspected high-risk pregnant women will lead to failure to identify and diagnose about $30 \%$ of hypothyroid and $69 \%$ of hyperthyroid women. Similarly, in China, research conducted $[10,23]$ shows that A case-finding strategy for screening thyroid function in the high-risk group would miss about $81.6 \%$ of pregnant women with hypothyroidism and $80.4 \%$ pregnant women with hyperthyroidism.

Thyroid screening is recommended for every pregnant woman in China, because they recognised the potential danger this condition posse to pregnancy outcome, and saw the need to be proactive toward preventing such from occurring. However, no published study evaluates this policy's outcome towards the overall wellbeing and outcome of pregnancies.

As physicians, the need for proper patients care cannot be overemphasised. Looking back at the policy and decision to implement screening for all women who intend to get pregnant and pregnant women, there is a need to evaluate this policy properly, the benefit and possible need for modification in management guideline in some areas, if need be. This study could be one way of accessing the efficacy of the diagnostic and management guideline for thyroid dysfunction in pregnancy.

\section{Methods}


This is a retrospective cohort study conducted at the Second Affiliated Hospital of Chongqing Medical University, using patients record from both the new hospital and old hospital. Second Affiliated Hospital of Chongqing Medical University is one of the public hospitals affiliated to Chongqing Medical University for training doctors in various medical fields. The hospital was established in 1892, and to date, the hospital is sited in two districts, Yuzhong and Nanaan district, with an average delivery of 3,000 babies annually.

Approval from the hospital management to make use of the patient's data for this research was granted. Data was collected from the Obstetrics and Gynecology department hospital's computer database, including outpatient medical history and inpatients management until delivery.

In this study, patients were managed for thyroid dysfunction, in line with the Second Affiliated Hospital of Chongqing Medical University guideline for managing patients with thyroid dysfunction before and during pregnancy. This guideline was set by the Compilation Committee of guidelines for diagnosis and treatment of thyroid diseases in pregnancy and postpartum, consisting of Endocrinology branch of Chinese Medical Association and Perinatal medicine branch of Chinese Medical Association.

This study was tailored towards patients who sought medical attention in the Second Affiliated Hospital of Chongqing Medical University, screened, diagnosed and medically managed, either by intervention or observation, for pregestational or gestational thyroid dysfunction in-line with the hospital guideline from 2016-2018. Included in this study are single and twin pregnancies, assisted and non-assisted pregnancies. This study's pregnancy outcome was divided into two, complications and non-complication, from conception to delivery. The complications examined are gestational hypertension, preeclampsia/eclampsia, abortion/miscarriage, preterm delivery, stillbirth, and intrauterine growth restriction. Other variables like gestational age, parity, gravida, age, and delivery mode were also included. Women with incomplete data and inadequate follow-up during pregnancy for proper history were excluded in this study's analysis and result.

In this study, the first trimester (T1) starts from the $1^{\text {st }}$ week to $13^{\text {th }}$ week +6 days, $2^{\text {nd }}$ trimester (T2) starts from $14^{\text {th }}$ week to $27^{\text {th }}$ week +6 days, and the $3^{\text {rd }}$ trimester (T3) starts from $28^{\text {th }}$ week to 42 nd week. The reference range used in the diagnosis of thyroid dysfunction in this study is trimester dependent and was adopted by Second Affiliated Hospital of Chongqing Medical University, and are as follow; TSH ( $\mu \mathrm{lU} / \mathrm{ml}) 0.35$ - 5.00 (Non-pregnant), 0.05 - 5.17 (first trimester), 0.39 - 5.22 (second trimester), 0.60 - 6.84 (third trimester); T4 (pmol/L) 9.5 - 24.5 (non-pregnant), 12.91 - 22.35 (first trimester), 9.81 - 17.26 (second trimester), 12.0 - 15.71(third trimester); T3 (pmol/L) 2.1 - 6.3. Abortion/miscarriage was defined as a pregnancy loss before 20 weeks' gestation. Stillbirth was defined as a pregnancy loss after 20 weeks' gestation. Preterm birth was a birth between $20+0$ weeks and $36+6$ weeks. Preeclampsia (PE) is defined as systolic blood pressure at $\geq 140 \mathrm{~mm} \mathrm{Hg}$ or diastolic blood pressure at $\geq 90 \mathrm{~mm} \mathrm{Hg}$ on at least two occasions measured 4 hours apart in previously normotensive woman and is accompanied by one or more of the following new-onset conditions at or after 20 weeks gestation: proteinuria; evidence of other maternal organ dysfunction; uteroplacental dysfunction [24]. Gestational hypertension is defined as a systolic blood pressure $140 \mathrm{mmHg}$ or more or diastolic blood pressure of $90 \mathrm{mmHg}$ or more, or both, on two occasions at least 4 hours apart after 20 weeks of gestation, in a woman with previously normal blood pressure [25]. The most widely used definition of IUGR is a foetus whose estimated weight is below the 10th percentile for its gestational age and whose abdominal circumference is below the 2.5 th percentile. IUGR was defined by a birth weight below the 10 th percentile

Participants were grouped according to the time they were diagnosed and managed, group 0 are those with history of thyroid dysfunction (G0), group 1 are those diagnose and managed at first trimester (G1), group 2 are those diagnosed and managed at second trimester (G2), group 3 are those diagnosed and managed at third trimester (G3).

The following set of patients were not included in this study; Non-pregnant women with thyroid dysfunction, post gestational thyroid dysfunction, pregnant foreigners(non-Chinese) diagnosed with pregestational or gestational thyroid dysfunction using the hospital's facility, and patients who were diagnosed with thyroid dysfunction without a complete medical record up to delivery. 
Statistical analysis, using SPSS package, was performed to compare outcome using the multiple logistic regression and binary logistic regression. The confidence interval $(\mathrm{Cl})$ at $95 \%$ was calculated. Level of significance was set at $p$-value $<0.05$. All analyses were adjusted for potential confounders such as maternal age.

\section{Results}

A total of 10,108 pregnant women were screened, of which 774(7.66\%) patients were diagnosed with thyroid dysfunction during their prenatal care from 2016-2018. Of the total number of diagnosed pregnant women, $448(4.43 \%)$ were diagnosed with subclinical hypothyroidism, 259(2.56\%) were diagnosed with hypothyroidism, 36(0.36\%) were diagnosed with hyperthyroidism, and 26(0.26\%) were diagnosed with other thyroid diseases. Of the 774 women, only 724 have a complete history from prenatal to postpartum and were considered for the analysis, excluding 50 women with incomplete data. Women with complete data, diagnosed and managed for subclinical hypothyroidism, hypothyroidism, hyperthyroidism, and other thyroid dysfunction are 420,246, 32, and 26, respectively. 79(10.9\%) among these women diagnosed and managed for various thyroid dysfunction developed complication, $41(5.63 \%), 30(4.12 \%), 4(0.55 \%)$, and $4(0.55 \%)$ for subclinical hypothyroidism, hypothyroidism, hyperthyroidism, and other thyroid dysfunction respectively.

Out of 724 analysed cases, $189(26.1 \%)$ women have an existing history of thyroid dysfunction, $276(38.1 \%)$ women were diagnosed at their first trimester, 164(22.7\%) women were diagnosed at their second trimester, and 95(13.1\%) women were diagnosed at their third trimester. Among these women the following developed complication at the course of pregnancy; $21(11.1 \%)$ women with an existing history of thyroid dysfunction, 33(12\%) women among those diagnosed at their first trimester, 13(7.9\%) women among those diagnosed at their second trimester, and 12(12.6\%) women among those diagnosed at their third trimester. The descriptive analysis of the age distribution shows that the means age and standard deviation is $30.03 \pm 4.413$, with their age ranging from 18 to 47 . The mean $(95 \% \mathrm{Cl})$ gestational age at delivery of all women enrolled in this study was 38.7 (38.5-38.9) weeks.

To ascertain the outcome of the management approach employed for pregnant women with a history of thyroid dysfunction or diagnosed with thyroid dysfunction after conception, we analysed the data using multivariate logistic regression. There was no significant difference in pregnancy outcome among women who were diagnosed and managed of subclinical hypothyroidism P-0.876(OR.0.882, 95\% Cl 0.182-4.281), Hypothyroidism, P-0.781(OR.0.798, 95\% Cl 0.163-3.912), hyperthyroidism, $P-0.427(O R .2 .411,95 \% \mathrm{Cl} 0.274-21.197)$ with other thyroid dysfunction as reference. No significant difference was found among the four groups, G0, G1, G2, and G3 who were diagnosed with thyroid dysfunction at different trimester, P-0.992(OR-1.005, 95\% Cl-0.398-2.533), P-0.882(OR-0.938, 95\%Cl-0.401-2.195), P-0.199(OR 2.009, 95\% Cl 0.6935.823), respectively. Also, there was no significant difference in maternal age, parity, and gestational age of the foetus at delivery. However, there was a significant difference in gravida, $P-0.032(O R 1.368,95 \% \mathrm{Cl} 1.0281 .821)$ and normal delivery mode, $P-0.010$ (OR 2.521, 95\% Cl 1.246-5.100). As shown in table 5.

To examine the relationship of each complication among all the four groups with age, for subclinical Hypothyroidism and Hypothyroidism alone, we analysed this using a binary logistic regression. The result shows a significant difference in maternal age for abortion/miscarriage among patients diagnosed with subclinical hypothyroidism $P-0.018(O R$ 1.459, $95 \% \mathrm{Cl}$ 1.067-1.997) and a significant difference in $\mathrm{G} 2$ for intrauterine growth restriction among women diagnosed with Hypothyroidism, $P-0.048(O R-0.152,95 \% \mathrm{Cl}$ 0.024-0.981) as shown in table 6.

\section{Discussion}

This study aimed to evaluate the outcome of screening and management of pregnant women diagnosed with various thyroid dysfunctions at different gestational ages in line with the hospital guideline. A total of 10,108 pregnant women were attended to and screened for thyroid dysfunction at Second Affiliated Hospital of Chongqing Medical University from 2016 - 2018, of which 774 women were diagnosed with thyroid dysfunction of various type. 448(4.4\%) of the pregnant population were diagnosed with subclinical hypothyroidism which is within the prevalence range of $3 \%-8 \%$ [26] and $2 \%$ - $5 \%$ [27], but far lesser 
than what is obtained in a study by Siobhan D et al.,15\% - 28\% [28], and Jianxia F. et al., 13.6\% [29]. Hypothyroid women were $259(2.6 \%)$ which appears to be higher than the typical pregnancy prevalence value of $0.3 \%-0.5 \%[2,27]$ and as well higher than the prevalence rate of 1.0\% [30]. Despite high cut-off values set by the Second Affiliated Hospital of Chongqing Medical University for diagnosis of hypothyroidism, the number of pregnant women with hypothyroidism appeared to be higher than what was obtained in other studies. This increase could be due to an increased Assisted Reproduction Technique application which has increased the incidence of multiply pregnancy, a risk factor to hypothyroidism [31]. Hyperthyroidism among pregnant women in this study is, $36(0.4 \%)$, which is in line with $0.1-0.4 \%$ [2]. Due to uncommon and infrequent occurrence of other thyroid dysfunction like subclinical hyperthyroidism, hypothyroxinemia, TPOA, and other thyroid dysfunction, which could lead to insignificant and inadequate sample size, hence they were grouped together with a total of $26(0.3 \%)$.

Various authors have suggested the time of diagnosis and management of thyroid dysfunction to be a contributing factor toward the pregnancy outcome as some believe that early diagnosis and management improves the outcome of the pregnancy on both the mother and baby [27], this might be true when it comes to thyroid dysfunction and its effect on Intelligent Quotient (IQ) [32,33], but it is not within the scope of this study. The time of diagnosis and initiation of appropriate management following the hospital guideline was noted, and the patients were divided into four groups. We analysed the relationship between thyroid dysfunction in all the four groups with pregnancy outcome, and there was no significant difference in all the groups as it relates to thyroid dysfunction and pregnancy outcome. This shows that pregnancy outcomes for women who developed thyroid dysfunction at any pregnancy stage are favourable when immediately identified and managed accordingly.

To ascertain the relationship between modes of delivery for thyroid dysfunction women in all the groups, we observed a significant difference in normal vagina delivery. The significance of normal vagina delivery could be a reflection of minimal complication. However, more than $60 \%$ of caesarean sections (CS) performed in the hospital this study was conducted are elective c-section; hence, it cannot be said to measure the efficacy of the management outcome. Gravida is seen as a possible risk factor for complication among pregnant women with thyroid dysfunction, as shown in this study. For every increase in pregnancy (gravida), there is 0.368 risk of developing complication during pregnancy. Most studies do not show the relationship between gravida and pregnancy outcome among women managed for thyroid dysfunction during pregnancy. This unidentified influence of gravida could be the reason why there is no special consideration among multigravida women, grand-multigravida, multiparous, and grand-multiparous in the management guideline. Primigravida and multigravida are risk factors to certain unfavourable pregnancy outcome like preeclampsia, dystocia [34,35], and abnormal foetal presentation, uterine atony, postpartum haemorrhage, placenta previa, and amniotic fluid embolism [36], respectively, and should be considered while managing pregnant women diagnosed with thyroid dysfunction.

Individual analysis of each complication for women diagnosed and managed of subclinical Hypothyroidism and Hypothyroidism at various gestational ages $(\mathrm{G} 0, \mathrm{G} 1, \mathrm{G} 2, \mathrm{G} 3)$ with maternal age showed a positive association between maternal age and abortion/miscarriage among women diagnosed and managed for subclinical hypothyroidism. Increase in maternal age by 1 year increases the risk of abortion/miscarriage by 0.459 among women diagnosed and managed in-line with the guideline for subclinical hypothyroidism. The guideline does not recommend intervention with Levothyroxine (LT4) for pregnant women diagnosed with subclinical hypothyroidism, whose TSH $>2.5 \mathrm{mu} / \mathrm{L}$ and higher than the lower limit of the specific reference range (or $0.1 \mathrm{mu} / \mathrm{L}$ ). When TPOAb is positive, TSH is monitored. When TPOAb is negative, there is no need to monitor TSH (Recommendation D) [37,38]. Despite efforts made to manage pregnant women diagnosed with subclinical hypothyroidism with optimum seriousness by deciding the therapeutic drugs, therapeutic targets and monitoring frequency of pregnant women with $\mathrm{SCH}$ to be the same as those of pregnant women with hypothyroidism, these lapses which perhaps explain the reason for the significant difference in abortion/miscarriage for aged women could be as a result of the recommendation that allows the therapeutic dose of LT4 to be less than that of clinical hypothyroidism during pregnancy. Depending on the degree of TSH elevation, different doses of LT4 may be given to initiate treatment (Recommendation A) $[37,38]$. This degree of freedom to choose, when to and how to, might sabotage the goals and objectives of SCH management in pregnancy. The mean age of 30.03 of women in this study indicates a drift towards advancing maternal age pregnancy in society and should be considered while drawing policy for thyroid dysfunction management. The significance of this outcome 
shows there is need for improvement in the management approach to curb the incidence of abortion/miscarriage among pregnant women with subclinical hypothyroidism as they advance in age.

Also, there appears to be a relationship between hypothyroidism and intrauterine growth restriction (IUGR) among women diagnosed and managed in their second trimester (G2). Our result shows for every increase in gestational age by one week, the chances of developing intrauterine growth restriction during second trimester increases by 0.848 . This complication could be attributed to delayed antenatal care, as most pregnant women commence antenatal in their second trimester. It is expected that the effect of pregnancy on thyroid function will be minimal in the second trimester due to decrease in demand by the foetal system as the foetal thyroid gland develops and synthesise thyroid hormone need by the body (6).

Limitations in this study include insufficient sample size for each thyroid dysfunction, which leads to the combination of thyroiditis, thyrotoxicosis, and other thyroid dysfunction. We do not compare the outcome of management of these women with a control group. This study is not a multicentre study, hence the reason for sampling bias. As a population-level retrospective analysis, our study can provide evidence of association only, not of causation. Over 50 women were excluded from the study due to incomplete follow-up records and could be considered a considerable sample size capable of influencing the outcome of the study.

\section{Conclusion}

The screening of thyroid dysfunction among pregnant women and intending pregnant women has led to early identification of pregnant women in need of close monitoring and thyroid dysfunction management. The overall pregnancy outcome of women diagnosed and managed for thyroid dysfunction in line with the guideline for diagnosis and treatment of thyroid diseases in pregnancy and postpartum which is set by Endocrinology branch of Chinese Medical Association and Perinatal medicine branch of Chinese Medical Association shows a promising result as less complication is observed. However, more attention is needed to properly manage subclinical hypothyroidism in pregnancy, hence curbing the incidence of abortion/miscarriage. Hypothyroidism in second trimester could lead to intrauterine growth restriction when not adequately managed. Multigravida could increase the risk of complication among pregnant women with thyroid dysfunction.

\section{Declarations}

Funding: There was no funding from any institution or organisation for this study.

Conflict of interest/competing interest: The authors declare that they have no conflict of interest.

Availability of data and material

Code availability: Not applicable

Author's contribution: This study was conducted and written by Samuel Obiegbusi, data collection by Deng Mingyu and Chidera Obiegbusi, statistical analysis by Yin Yang, translation of management guideline from Chinese to English by Xinru Deng, and Prof. Xiao Jing Dong supervised the study. All authors read and approved the final manuscript.

Ethics approval: The management approved this study and the use of patients record and medical information in the Second Affiliated Hospital of Chongqing Medical University.

Consent to participate: Not applicable

Consent for publication: All authors are in support of the submission of this study for publication.

\section{References}


1. Alexander EK, Pearce EN, Brent GA, Brown RS, Chen H, Dosiou C, Grobman WA, Laurberg P, Lazarus JH, Mandel SJ, Peeters RP, Sullivan S (2017) 2017 guidelines of the American Thyroid Association for the diagnosis and management of thyroid disease during pregnancy and the postpartum. Thyroid 27(03):315-389

https://pubmed.ncbi.nlm.nih.gov/28056690/

2. Dotun A Ogunyemi (2016) Autoimmune thyroid disease and pregnancy. Medscape https://emedicine.medscape.com/article/261913-overview

3. Allan WC, Haddow JE, Palomaki GE, Williams JR, Mitchell ML, Hermos RJ, Faix JD, Klein RZ (2000) Maternal thyroid deficiency and pregnancy complications: implications for population screening. J Med Screen 7:127-130. https://pubmed.ncbi.nlm.nih.gov/11126160/

4. Hirsch D, Levy S, Nadler V, Kopel V, Shainberg B, Toledano Y (2013) Pregnancy outcomes in women with severe hypothyroidism. Eur J Endocrinol 169(03):313-320

5. Pregnancy and Thyroid Disease. Am Thyroid Assoc. https://www.thyroid.org/thyroid-disease-pregnancy/

6. Michel P (2014) Human fetal thyroid function, Paediatric Thyroidology 26:17-25. https://pubmed.ncbi.nlm.nih.gov/25231441/

7. Neale DM, Cootauco AC, Burrow G (2007) Thyroid disease in pregnancy. Clin Perinatol. 34 (4):543-57, v-vi. doi: 10.1016/j.clp.2007.10.003. https://pubmed.ncbi.nlm.nih.gov/18063104/

8. Parizad NM, Badfar G, Shohani M, Rahmati S, YektaKooshali MH, Abbasalizadeh S, Soleymani A, Azami M (2017) The relation of maternal hypothyroidism and hypothyroxinemia during pregnancy on preterm birth: An updated systemic review and meta-analysis. Int J Reprod BioMed 15:543-552.

9. Dhanwal DK, Bajaj S, Rajput R, Subramaniam KVA, Chowdhury S, Bhandari R, Dharmalingam M, Sahay R, Ganie A, Kotwal N, Shriram U (2016) Prevalence of Hypothyroidism in pregnancy: An epidemiological study from 11 cities in 9 states of India. Indian J Endocrinol Metab 20: 387-390. DOI:10.4103/2230-8210.179992

10. Lazarus J, Brown RS, Daumerie C, Hubalewska-Dydejczyk A, Negro R, Vaidya B. (2014) 2014 European thyroid association guidelines for the management of subclinical hypothyroidism in pregnancy and in children. Eur Thyroid 3:7694. https://pubmed.ncbi.nlm.nih.gov/25114871/

11. Li C, Shan Z, Mao J, Wang W, Xie X, Zhou W, Li C, Xu B, Bi L, Meng T (2014) Assessment of Thyroid function during firsttrimester pregnancy: What is the rational upper limit of serum TSH during the first trimester in Chinese pregnant women? J Clin Endocrinol Metab. 99(1):73-79. DOI: 10. 1210/jc. 2013-1674 https://pubmed.ncbi.nlm.nih.gov/24276458/

12. Erik KA, Elizabeth NP, Gregory AB, Rosalind SB, Herbert C, Chrysoula D, William AG, Peter L, et al. (2017) 2017 Guidelines of the American Thyroid Association for the Diagnosis and Management of Thyroid Disease during Pregnancy and the Postpartum. Thyroid 27(3): 315-389. doi:10.1089/thy.2016.0457

13. Roberto N, Roberto A, Enrico P, Rinaldo G, Franco G, Vincenzo T, Dan AN et al.(2018) A 2018 Italian and Romanian Survey on Subclinical Hypothyroidism in Pregnancy. Eur Thyroid J 7(6):294-301. https://pubmed.ncbi.nlm.nih.gov/30574459/

14. Zhang D, Cai K, Wang G, Xu S, Mao X, Zheng A, Liu C, Fan K (2019) Trimester-specific reference ranges for thyroid hormones in pregnant women. Medicine 98(4):P.e14245 https://doi.org/10.1097/MD.0000000000014245

15. Chinese Society of Endocrinology, Chinese Medical Association, Chinese Society of Perinatology (2019) Guideline on diagnosis and management of thyroid disease during pregnancy and postpartum ( $2^{\text {nd }}$ edition) Chin J Perinat Med, 35: 8

16. Li Jia, Teng Weiping, Shan Zhongyan, et al. (2008) Specific gestational reference range of TSH and T4 in Han nationality of China [J]. Chinese Journal of Endocrinology and metabolism. 24(6):605-608. DOI: 10.3321/j.issn:10006699.2008.06.004.

17. Shen FX, Xie ZW, Lu SM, et al. (2014) Gestational thyroid reference intervals in antibody-negative Chinese women. Clin Biochem, 47(7-8):673-675. DOI: 10.1016/j.clinbiochem.2014.02.03

18. Liu J, Yu X, Xia M, et al. (2017) Development of gestation-specific reference intervals for thyroid hormones in normal pregnant Northeast Chinese women: What is the rational division of gestational stages for establishing reference intervals for pregnant women? Clin Biochem. 50(6):309-317. DOI: 10.1016/j.clinbiochem.2016.11.036. 
19. Yan YQ, Dong ZL, DOng L, et al. (2011) Trimester and method-specific reference intervals for thyroid tests in pregnant Chinese women: methodology, euthyroid definition and iodine status can influence the setting of reference intervals. Clin Endocrinol (Oxf), 74(2):262-269. DOI: 10.1111/j.1365-2265.2010.03910.x.

20. Zhang Ning, Yan Suwen, Xu Bin, et al. (2013) Establishing the role of regional, gestational age and method specific thyroid hormone reference range in the evaluation of thyroid function during pregnancy [J]. China Journal of eugenics and genetics 21(3):57-59,83.

21. Sun Qiujin, Yan Wei (2015) Establishment and analysis of reference interval for thyroid screening during pregnancy: International Journal of Laboratory medicine 36(20):3013-3015. DOI: 10.3969/j.issn.1673-4130.2015.20.039

22. Vaidya B, Anthony S, Bilous M, Shields B, Drury J, Hutchison S, Bilous R (2007) Detection of thyroid dysfunction in early pregnancy: universal screening or targeted high-risk case finding? J Clin Endocrinol Metab 92:203-207 https://pubmed.ncbi.nlm.nih.gov/17032713/

23. Teng W, Shan Z, Wang S, Li J, Zhu L, Zhou J, Mao J, Yu X, Li J, Chen Y et al.(2011) The prevalence of thyroid disorders during early pregnancy in China: the benefits of universal screening in the first trimester of pregnancy. Eur $J$ Endocrinol. 164:263-268. https://pubmed.ncbi.nlm.nih.gov/21059864/

24. Poon LC, Shennan A, Hyett JA, Kapur A, Haar E, Divakar H, McAuliffe F, Silva-Costa F, Dadelszen P, Mclntyre HD, Kihara AB et al. (2019) The International Federation of Gynecology and Obstetrics (FIGO) initiative on preeclampsia: A pragmatic guide for first-trimester screening and prevention. Int $J$ Gynaecol Obstet. 145(S1):1-33.

https://pubmed.ncbi.nlm.nih.gov/31111484/

25. ACOG Practice Bulletin, Number 222 (2020). Gestational Hypertension and Preeclampsia: Obstet Gynecol. 135(6):e237e260. doi:10.1097/AOG.0000000000003891. https://pubmed.ncbi.nlm.nih.gov/32443079/

26. Rao M, Zeng Z, Zhao S, Tang L, et al.(2018) Effect of levothyroxine supplementation on pregnancy outcomes in women with subclinical hypothyroidism and thyroid autoimmunity undergoing in vitro fertilisation/intracytoplasmic sperm injection: an updated meta-analysis of randomised controlled trials. Reprod Biol Endocrinol 16:92 https://doi.org/10.1186/s12958-018-0410-6

27. Rakesh S and Sri-Nagesh V (2012) Hypothyroidism in Pregnancy. India Journal of Endocrinology and Metabolism 16(3):364-370 https://www.ncbi.nlm.nih.gov/pmc/articles/PMC3354841/\#ref14

28. Siobhan D and Ahraaz W (2017) Subclinical Hypothyroidism in pregnancy.Canadian Medical Association Journal 189(28):E941 https://www.ncbi.nlm.nih.gov/pmc/articles/PMC5515647/

29. Jianxia F, Yong Z, Chen Z, Mirjana B, Xi Y, Robin P, He-Feng H, Tim K (2019) Persistency of Thyroid Dysfunction From Early to Late Pregnancy. Thyroid 29(10):1475-1484 https://pubmed.ncbi.nlm.nih.gov/31347461/

30. Teng W, Shan Z, Patil-Sisodia K, et al.(2013) Hypothyroidism in Pregnancy. Lancet Diabetes Endocrinol 1(3):228-237. DOI: 10.1016/S2213-8587(13)70109-8.

31. Hall JG (1996) Twinning mechanisms and genetic implications. Curr Opin Genet Dev. 6:343-347 https://doi.org/10.1016/S0959-437X(96)80012-8

32. Li Y, Shan Z, Teng W, et al.(2010) Abnormalities of maternal thyroid function during pregnancy affect neuropsychological development of their children at 25-30 months. Clin Endocrinol (oxf). 72(6):825-829. DOI: 10.1111/j.13652265.2009.03743.x.

33. Haddow JE, Palomaki GE, Allan WC, et al.(1999) Maternal thyroid deficiency during pregnancy and subsequent neuropsychological development o the child. N. Engl J Med. 341(8):549-555. DOI: 10.1056/NEJM199908193410801.

34. Emily Bartsch, Karyn E Medcalf, Alison L Park, Joel G Ray (2016) Clinical risk factors for preeclampsia determined in early pregnancy: systematic review and meta-analysis of large cohort studies, BMJ. 353:i1753. doi:10.1136/bmj.i1753

35. Kjaergaard H, Olsen J, OAttesen B, et al. (2009) Incidence and outcomes of dystocia in the active phase of labor in term nulliparous women with spontaneous labor onset. Acta Obstet Gynecol Scand. 88 (4):402-407.

36. Waldenstrom U, Ekeus C (2017) Risk of labor dystocia increases with maternal age irrespective of parity: a populationbased register study. Acta Obstet Gynecol Scand. 96 (9):1063-1069. doi: 10.1111/aogs.13167. 
37. Endocrinology branch of Chinese Medical Association and Perinatal medicine branch of Chinese Medical Association. (2019) Guidelines for diagnosis and treatment of thyroid diseases in pregnancy and postpartum (2nd Ed). Chinese Journal of perinatal Medicine 22(8).

38. Endocrine Society, American Association of Clinical Endocrinologists, Asia and Oceania Thyroid Association, et al. management of thyroid dysfunction during pregnancy and postpartum: An Endocrine Society Clinical Practice Guideline. Thyroid 2007;17(11):1159-1167. DOI: 10.1089/thy.2017.1523

\section{Tables}

Table 1: Second Affiliated Hospital of Chongqing Medical University thyroid trimester reference range.

\begin{tabular}{|lllll|}
\hline & Non-pregnant & First trimester & Second trimester & Third trimester \\
\hline TSH $(\mu \mathrm{lU} / \mathrm{ml})$ & $0.35-5.00$ & $0.05-5.17$ & $0.39-5.22$ & $0.60-6.84$ \\
\hline $\mathrm{T} 3 \mathrm{pmol} / \mathrm{L}$ & $2.1-6.3$ & & & \\
\hline $\mathrm{T} 4 \mathrm{pmol} / \mathrm{L}$ & $9.5-24.5$ & $12.91-22.35$ & $9.81-17.26$ & $12-15.71$ \\
\hline TSH: Thyroid-stimulating hormone, T3: Triiodothyronine, T4: Thyroxine & \\
\hline
\end{tabular}

\begin{tabular}{|c|c|c|c|c|}
\hline & Non-pregnant & First trimester & Second trimester & Third trimester \\
\hline TSH(mIU/L) & & 0.02 to 3.78 & 0.47 to 3.89 & 0.55 to 4.91 \\
\hline FT4pmol/L & & 13.93 to 26.49 & 12.33 to 19.33 & 11.38 to 19.21 \\
\hline TT4 nmol/L & & 103.39 to 319.43 & 92.28 to 234.88 & 83.54 to 258.12 \\
\hline
\end{tabular}




\begin{tabular}{|c|c|c|c|c|c|c|c|}
\hline \multicolumn{4}{|c|}{$\mathrm{TSH}(\mathrm{mU} / \mathrm{L})$} & \multicolumn{4}{|l|}{ FT4 (pmol/ L) } \\
\hline $\begin{array}{l}\text { Reagent } \\
\text { Company }\end{array}$ & $\begin{array}{l}\text { First } \\
\text { trimester }\end{array}$ & $\begin{array}{l}\text { Second } \\
\text { trimester }\end{array}$ & $\begin{array}{l}\text { Third } \\
\text { trimester }\end{array}$ & $\begin{array}{l}\text { First } \\
\text { trimester }\end{array}$ & $\begin{array}{l}\text { Second } \\
\text { trimester }\end{array}$ & $\begin{array}{l}\text { Third } \\
\text { trimester }\end{array}$ & method \\
\hline DPC & $0.13 \sim 3.93$ & $0.26 \sim 3.50$ & $0.42 \sim 3.85$ & $12.00 \sim 23.34$ & $11.20 \sim 21.46$ & $9.80 \sim 18.20$ & $\begin{array}{l}\text { Chemiluminescence } \\
\text { immunoassay law }\end{array}$ \\
\hline Abbott & $0.07 \sim 3.38$ & $0.34 \sim 3.51$ & $0.34 \sim 4.32$ & $11.30 \sim 17.80$ & $9.30 \sim 15.20$ & $7.90 \sim 14.10$ & $\begin{array}{l}\text { Chemiluminescence } \\
\text { immunoassay law }\end{array}$ \\
\hline Roche & $0.09 \sim 4.52$ & $0.45 \sim 4.32$ & $0.30 \sim 4.98$ & $13.15 \sim 20.78$ & $9.77 \sim 18.89$ & $9.04 \sim 15.22$ & $\begin{array}{l}\text { Electrochemical } \\
\text { immunoassay law }\end{array}$ \\
\hline Bayer & $0.03 \sim 4.51$ & $0.05 \sim 4.50$ & $0.47 \sim 4.54$ & $11.80 \sim 21.00$ & $10.60 \sim 17.60$ & $9.20 \sim 16.70$ & $\begin{array}{l}\text { Chemiluminescence } \\
\text { immunoassay law }\end{array}$ \\
\hline Beckman & $0.05 \sim 3.55$ & $0.21 \sim 3.31$ & $0.43 \sim 3.71$ & $9.01 \sim 15.89$ & $6.62 \sim 13.51$ & $6.42 \sim 10.75$ & $\begin{array}{l}\text { Chemiluminescence } \\
\text { immunoassay law }\end{array}$ \\
\hline DiaSorin & $0.02 \sim 4.41$ & $0.12 \sim 4.16$ & $0.45 \sim 4.60$ & $8.47 \sim 19.60$ & $5.70 \sim 14.70$ & $5.20 \sim 12.10$ & $\begin{array}{l}\text { Chemiluminescence } \\
\text { immunoassay law }\end{array}$ \\
\hline Tosoh & $0.09 \sim 3.99$ & $0.56 \sim 3.94$ & $0.56 \sim 3.94$ & $10.42 \sim 21.75$ & 7.98 18.28 & 7.33 15.19 & $\begin{array}{l}\text { Chemiluminescence } \\
\text { Immunoassay }\end{array}$ \\
\hline \multicolumn{8}{|c|}{$\begin{array}{l}\left.\text { Copied from Guideline on diagnosis and management of thyroid disease during pregnancy and postpartum ( } 2^{\text {nd }} \text { edition }\right) \\
\text { Ad Hoc writing committee for guidelines on diagnosis and management of thyroid disease during pregnancy and } \\
\text { postpartum; Chinese society of Endocrinology, Chinese Medical Association; Chinese Society of Perinatology; Chinese } \\
\text { Medical Association. }\end{array}$} \\
\hline \multicolumn{8}{|c|}{ This table was translated from the original Chinese version to English version. } \\
\hline
\end{tabular}


Table 4. The recommended guideline for the management of Subclinical hypothyroidism, hypothyroidism, Positive thyroid autoantibodies

\section{SERIAL RECOMMENDATION NUMBER}

RECOMMENDATION STRENGTH

\section{The reference range of related index of thyroid function during pregnancy}

For thyroid dysfunction diagnosis during pregnancy, the reference range of serum

Recommendation A middle and third trimester) should be established in this unit or in this area.

1.2 Adopt the method recommended by the American Institute of Clinical Biochemistry $\triangle N A C B \otimes$ to establish the reference range. Pregnant women with a moderate amount of

Recommendation A iodine, singletons, previous thyroid diseases, negative thyroid autoantibodies and no goitre were selected. The reference range was 2.5 to 97.5 percentiles.

2

\section{Clinical hypothyroidism during pregnancy (Hypothyroidism)}

2.1 The diagnostic criteria of clinical hypothyroidism during pregnancy are as follows:

Recommendation A serum TSH > upper limit of the pregnancy-specific reference range, serum FT4 < lower limit of the pregnancy reference range.

2.2 If the pregnancy-specific reference range of TSH cannot be obtained, the tangent point of the upper limit of TSH in early pregnancy can be obtained by the following two methods: the value obtained by reducing the upper limit of TSH reference range by $22 \%$ or $4.0 \mathrm{mU} / \mathrm{L}$ in the general population.

2.3 During pregnancy, clinical hypothyroidism impairs the neurointellectual development of offspring and increases the risks of preterm delivery, abortion, low birth weight, stillbirth, and high blood pressure during pregnancy, which must be treated.

Recommendation A

\section{Treatment}

The goal of treating clinical hypothyroidism in pregnancy is

2.4 During pregnancy, the treatment goal of clinical hypothyroidism is to control TSH at the lower 1 / 2 of the pregnancy-specific reference range. If the pregnancy-specific reference range cannot be obtained, the serum TSH can be controlled at 2. Less than $5 \mathrm{mU} / \mathrm{L}$. Once clinical hypothyroidism during pregnancy is diagnosed, treatment should be started immediately to achieve the above treatment goal as soon as possible.

2.5 Clinical hypothyroidism during pregnancy was treated with LT4. There is no need for LT3 or dry thyroid tablets.

Recommendation B

LT3 or dify thy

2.6 After clinical hypothyroidism women are suspected or diagnosed with pregnancy, the replacement dose of LT4 needs to be increased by $20 \%$ to $30 \%$. Adjust the dose of TSH in time according to the treatment target of serum LT4.

$2.7 \quad$ Thyroid function was measured every $2 \sim 4$ weeks in women with clinical hypothyroidism in the first half of pregnancy. When serum TSH is stable, it can be detected every $4 \sim 6$ weeks.

2.8 The postpartum dose of LT4 in pregnant women with clinical hypothyroidism should be adjusted to the pre-pregnancy level, and the thyroid gland function should be reexamined six weeks after delivery to guide the adjustment of LT4 dose. planning any pregnancy.

\section{3}

\section{Subclinical hypothyroidism during pregnancy $(\mathrm{SCH})$} of the pregnancy-specific reference range, and serum FT4 is within the reference range of pregnancy specificity.

Recommendation A

Recommendation A

Recommendation B

Recommendation A

Recommendation A

Recommendation A

Recommendation I 
b $\quad \mathrm{TSH}>2.5 \mathrm{mU} / \mathrm{L}$ and lower than the upper limit of the pregnancy-specific reference

Recommendation B norm (or $4.0 \mathrm{mU} / \mathrm{L}$ ), with TPOAb positive, LT4 therapy should be considered.

c $\quad \mathrm{TSH}>2.5 \mathrm{mU} / \mathrm{L}$ and lower than the upper limit of the pregnancy-specific reference

Recommendation D norm (or $4.0 \mathrm{mU} / \mathrm{L}$ ). TPOAb is negative. LT4 therapy is not considered.

d $\quad T S H<2.5 \mathrm{mU} / \mathrm{L}$ and higher than the lower limit of pregnancy-specific reference norm

Recommendation D (or $0.1 \mathrm{mU} / \mathrm{L}$ ), is not recommended for LT4 therapy). When TPOAb is positive, TSH needs to be monitored. When TPOAb negative, no need for monitoring

3.3 The therapeutic drugs, therapeutic targets and monitoring frequency of SCH during pregnancy are the same as those of clinical hypothyroidism during pregnancy. The

Recommendation A therapeutic dose of LT4 may be less than that of clinical hypothyroidism during pregnancy. Different doses of LT4 can be given initial treatment according to the degree of TSH elevation.

3.4 Postpartum withdrawal of LT4 may be considered in patients with $\mathrm{SCH}$, diagnosed during pregnancy, and serum TSH levels can be evaluated six weeks after delivery.

Recommendation B

$4 \quad$ Simple hypothyroidism during pregnancy

4.1 When the level of serum FT4 is lower than the lower limit of the pregnancy-specific reference range and the serum TSH is normal, hypothyroidism can be diagnosed.

4.2 There is insufficient evidence for LT4 intervention in uncomplicated hypothyroidism to

Recommendation C improve adverse pregnancy outcome and neurointellectual development damage in offspring. This finger South neither recommends nor opposes LT4 treatment in early pregnancy.

4.3 It is recommended to determine the causes of hypothyroidism, such as iron deficiency

Recommendation A iodine deficiency or iodine excess, and treat the causes.

\section{$5 \quad$ Positive thyroid autoantibodies during pregnancy}

5.1 Positive thyroid autoantibodies mean that the titer of TPOAb or TgAb exceeds the upper limit of the reference range provided by the kit. Simple positive thyroid

Recommendation A autoantibodies are not accompanied by abnormal serum TSH, which is also called positive thyroid autoantibodies in patients with normal thyroid function. should be monitored for serum TSH, every four weeks to the end of the second trimester after a definite pregnancy.

5.3 The use of LT4 in treating pregnant women with normal thyroid function, positive TPOAb and a history of unexplained abortion may be beneficial with low risk. It can

Recommendation B be treated with LT4 at the beginning of $25 \sim 50 \mu \mathrm{g} / \mathrm{d}$.

5.4 Selenium supplementation is not recommended for TPOAb positive women during

Recommendation D pregnancy.

\section{$6 \quad$ Postpartum thyroiditis (PPT)}

6.1 PPT will be onset within one year after parturition. The typical cases experienced three

Recommendation A stages, thyrotoxicosis, hypothyroidism and recovery. Atypical cases can only show thyrotoxicosis or hypothyroidism. Women with positive TPOAb in early pregnancy have an increased risk of developing PPT.

6.2 All patients with depression, including postpartum depression, should be screened for thyroid function.

Recommendation B

Patients with PPT thyrotoxicosis are not treated with Antithyroid drugs (ATD). Beta-
blockers can relieve symptoms, use the minimum dose and shorten the treatment

Patients with PPT thyrotoxicosis are not treated with Antithyroid drugs (ATD). Beta-
blockers can relieve symptoms, use the minimum dose and shorten the treatment course as much as possible. 

weeks until thyroid function normalise.

6.6 After continuous treatment for 6-12 months during Hypothyroidism, LT4 began to decrease gradually. If the patient needs breastfeeding at this time, do not reduce the

Recommendation C dose of LT4.

6.7 More than $20 \%$ of PPT patients will develop permanent hypothyroidism. Serum TSH needs to be tested every year after the onset of the disease. Early detection of permanent hypothyroidism and treatment is needed.

\section{$7 \quad$ Thyrotoxicosis during pregnancy}

7.1 Serum TSH in early pregnancy is lower than the lower limit of the pregnancy-specific reference range (or $0.1 \mathrm{mU} / \mathrm{L}$ ), ) suggesting that there may be thyrotoxicosis. Medical

Recommendation A history and physical examination should be asked in detail, and T4, T3, TRAb and TPOAb should be further determined. ${ }^{131}$ I uptake rate and radionuclide scanning are contraindicated.

7.2 Serum TSH is lower than the lower limit of the pregnancy-specific reference range (or

Recommendation A $0.1 \mathrm{mU} / \mathrm{L}), \mathrm{FT} 4>$ the upper limit of the pregnancy-specific reference range). After excluding hyperthyroidism, Transient thyrotoxicosis of pregnancy $₫ \mathrm{GTT}$ ) can be diagnosed.

7.3 GTT is associated with a high level of hCG secreted by the placenta. Supportive therapy is the primary treatment to correct dehydration and electrolyte disturbance.

Recommendation A ATD treatment is not recommended. If the condition requires, you can consider the use of $\beta$-receptor blockers.

7.4 Women with hyperthyroidism due to Graves disease had better chances of getting pregnant after the thyroid gland function is controlled to normal and stable and reduces the negative outcome of pregnancy.

7.5 Except for the rare case of simple fetal hyperthyroidism and the control of gestational hyperthyroidism, the combination of ATD and LT4 is not recommended. Because this will increase the therapeutic dose of ATD, leading to goitre and hypothyroidism in the fetus.

7.6 Pregnant women who are taking Methimidazole $\triangle \mathrm{MMI}$ or PropylthiouracilखPTU) can suspend ATD and immediately test for thyroid function and thyroid autoantibodies if the pregnancy test is positive. Decide whether or not to use drugs according to clinical manifestations and FT4 level.

Some patients can stop using ATD after the diagnosis of pregnancy is made. The decision to stop medication needs to consider medical history, goitre size, course of treatment, pre-pregnancy ATD dose, recent thyroid function results, TRAb levels and other clinical factors.

After withdrawal, if the FT4 is normal or close to normal, you can continue to stop the drug. Clinical evaluation and detection of TSH, FT4 or TT4 and T3 were performed every $1 \sim 2$ weeks. If FT4 remains normal, thyroid function can be monitored every 2-4 weeks in the middle and third trimester of pregnancy. According to the results of each evaluation, decide whether to continue to stop the drug for observation.

Recommendation B

Recommendation A



and TSH should be tested every $1 \sim 2$ weeks in the first trimester, every $2 \sim 4$ weeks in the middle and third trimester of pregnancy, and every $4 \sim 6$ weeks after reaching the target value.

7.9 In principle, no operation should be taken to treat hyperthyroidism during pregnancy. If necessary, the best time for thyroidectomy is in the second trimester of pregnancy.

Recommendation A

7.10 Serum TRAb was detected in pregnant women with Graves disease who had been

Recommendation A treated with radioactive iodine, surgery, or ATD in the early stage of pregnancy.

A If the serum TRAb is negative in the first trimester of pregnancy, there is no need to test again during pregnancy.

B If serum TRAb increases in the first trimester of pregnancy, it is recommended to detect it again at 18-22 weeks of pregnancy.

C If serum TRAb increases at 18-22 weeks of pregnancy or begins to use ATD, in the third trimester of pregnancy, it is necessary to detect serum TRAb, again to evaluate the necessity of fetal and neonatal monitoring.

7.11 For pregnant women whose maternal hyperthyroidism can not be controlled or stored

Recommendation A in high titer TRAb (3 times higher than the upper limit of the reference range) in the second half of pregnancy, fetal heart rate should be monitored from the second trimester, and fetal thyroid volume, growth and development, amniotic fluid volume and so on should be examined by ultrasound. The thyroid function of newborns with high-risk factors of hyperthyroidism should be closely monitored.

7.12 Patients with hyperthyroidism who are breastfeeding should weigh the advantages and disadvantages of using ATD,. ATD should be taken after each lactation.

Recommendation B

Recommendation A

Recommendation A lodine nutrition during pregnancy

\section{8}

8.1

When evaluating iodine nutrition of pregnant women, the ratio of single Urinary iodine concentration $\varangle \mathrm{UIC} \otimes$ to urinary creatinine $(\mu \mathrm{g} / \mathrm{g})$ was better than that of single UIC $(\mu$

Recommendation B $\mathrm{g} / \mathrm{L})$.

8.2 At least $250 \mu \mathrm{g}$ of iodine daily for pregnant, pregnant and lactating women.

Recommendation A

8.3 Formulate different iodine supplement strategies according to different regions. In iodine deficiency areas, if you eat iodised salt every day, there is no need to supplement iodine during pregnancy. If you do not eat iodised salt, you need to supplement an additional $150 \mu \mathrm{g}$ of iodine every day during pregnancy. Potassium iodide is suitable for iodine supplement (or multivitamins containing the same amount of potassium iodide). The best time to start supplementation is at least three months before pregnancy.

8.4 Daily iodine intake $>500 \mu \mathrm{g}$ during pregnancy and lactation is at risk of fetal hypothyroidism.

Recommendation C

\section{$9 \quad$ Thyroid nodules and thyroid carcinoma during pregnancy}

9.1 During pregnancy, patients with thyroid nodules should be asked for medical history in detail, physical examination should be improved, serum TSH should be determined, and neck ultrasound should be done.

A If the TSH level decreases and continues beyond 16 weeks of pregnancy, Fine needle aspiration (FNA) cytological examination of thyroid nodules may be postponed until postpartum. If postpartum TSH is still low, radionuclide scanning is feasible to evaluate thyroid nodule function without breastfeeding. sonographic characteristics of the nodules.

Recommendation A can be postponed until postpartum. If the thyroid nodule cytology is benign, no special monitoring is required during pregnancy. monitor the growth rate of the tumour. If the nodules remain stable in the second

Recommendation C 
trimester or found in the second half of pregnancy, the surgery may be postponed until postpartum.

9.4 Differentiated thyroid carcinoma (DTC) during pregnancy, with no operation in the early stage of pregnancy, should be reexamined by thyroid ultrasound every three

Recommendation C months to monitor the growth rate of the tumour. The goal of LT4 treatment is to control the serum TSH level in the range of $0.3 \sim 2.0 \mathrm{mU} / \mathrm{L}$.

9.5 If DTC continues to increase 24-26 weeks after, or lymph node metastasis occurs, surgical treatment is recommended.

9.6 The operation time of DTC should be in the second trimester of pregnancy. At this point, the risk of surgery to the mother and fetus is reduced.

9.7 The effect of newly diagnosed medullary or undifferentiated carcinoma during pregnancy on pregnancy is not clear. However, delayed treatment is likely to lead to adverse outcomes. Therefore, after evaluating all clinical factors, surgical treatment should be performed.

9.8 Patients with DTC should maintain the established goal of TSH inhibition after pregnancy. The serum TSH was detected regularly every $2 \sim 4$ Mondays until the 20th week of pregnancy. When TSH is stable, it can be detected every $4 \sim 6$ weeks.

Recommendation B

Recommendation B

Recommendation C

W.9 Women with a history of DTC treatment do not need an ultrasound and Tg monitoring
during pregnancy if there is no evidence of structural abnormalities of the disease

Recommendation A (whether there are suspicious cancer nodules on ultrasound) or biochemical (elevated Tg levels) before pregnancy. If thyroid cancer treatment is not effective, or if it is known to exist in recurrent or residual lesions, ultrasound and Tg monitoring should be performed during pregnancy.

10 Congenital Hypothyroidism (CH)

$10.1 \quad$ Neonatal $\mathrm{CH}$ screening should be carried out at $72 \mathrm{~h} \sim 7 \mathrm{~d}$ after birth. The TSH tangent point of heel blood (dry bloodstain on filter paper) is $10 \sim 20 \mathrm{mU} / \mathrm{L}$.

Recommendation B

10.2 Serum TSH, FT4 / TT4 was reexamined immediately in patients with positive

Recommendation A screening. Local laboratories determine the diagnostic criteria according to the reference values of their laboratories. Serum TSH $>9 \mathrm{mU} / \mathrm{L} \mathrm{FT} 4<0.6 \mathrm{ng} / \mathrm{dl}$ can be used as the diagnostic standard of $\mathrm{CH}$. It still needs to be combined with the results of the etiological examination of $\mathrm{CH}$.

10.3 $\mathrm{CH}$ treatment should be started within two months after birth, and the earlier the prognosis is, the better. The treatment goal is to maintain the $1 / 2$ level of serum TSH $<5 \mathrm{mU} / \mathrm{L} \mathrm{FT4}$ and TT4 in the reference range.

11 Screening of thyroid diseases during and before pregnancy

11.1 In high-risk pregnancy screening, $30 \% \sim 80 \%$ of hyperthyroidism, subclinical hyperthyroidism, hypothyroidism, and $\mathrm{SCH}$ missed diagnosis.

11.2 Cost-benefit analysis shows that screening the whole pregnant population is better than non-screening.

Recommendation A

than non-screening.

11.3

According to the national conditions of our country (China), this guide supports qualified domestic hospitals and maternal and child health departments to carry out thyroid disease screening for women in early pregnancy. Serum TSH, FT4 and TPOAb were selected as screening indexes. The screening time is chosen before eight weeks of pregnancy. It is best to screen before pregnancy.

12 Infertility and assisted reproduction and thyroid diseases

12.1 All women who are treated for infertility should monitor their serum TSH levels. Recommendation B

12.2 For $\mathrm{SCH}$ infertile women with negative thyroid autoantibodies (who did not receive assisted reproduction), there is insufficient evidence that LT4 treatment increases the pregnancy rate. However, the application of LT4 can prevent the development of SCH to clinical hypothyroidism after pregnancy, and the risk of low-dose LT4 treatment is low. It is recommended to give LT4 treatment to infertile women with $\mathrm{SCH}$, with an initial dose of $25 \sim 50 \mu \mathrm{g} / \mathrm{d}$.

Recommendation A 
The treatment target of TSH should be controlled below $2.5 \mathrm{mU} / \mathrm{L}$.

12.4 Because the thyroid gland function results obtained during controlled ovarian stimulation can not reflect the actual state of thyroid function, it is suggested that thyroid function should be tested $1 \sim 2$ weeks before and $1 \sim 2$ weeks after controlled ovarian stimulation.

12.5 For women who successfully conceived by controlled superovulation, it is recommended to treat those with elevated TSH. If TSH increases slightly for nonpregnant women who are not pregnant after controlled superovulation, TSH should be monitored every 2 to 4 weeks. The thyroid function of these women may return to a normal level.

12.6 For infertile women with normal thyroid function and TPOAb positive for assisted reproduction, there is insufficient evidence to improve the outcome of assisted

Recommendation C reproduction with LT4. However, for infertile women who have a history of abortion or recurrent abortion and perform assisted reproduction, they should weigh the advantages and disadvantages while choosing LT4 treatment. The initial dose of LT4 is $25 \sim 50 \mu \mathrm{g} / \mathrm{d}$.

\section{Rating of recommendation strength:}

A. Highly recommended. The evidence confirms that there are more advantages than disadvantages in improving health outcomes

B. Recommended. There is good evidence that improving health outcomes outweighs the disadvantages

C. Not recommended or opposed. Based on expert advice, or available evidence suggests that the pros and cons are close

D. Against recommendations. Because the evidence is not strong enough or does more harm than good for health outcomes

E. Against recommendations. Lack of evidence, or poor quality of evidence, or contradictory evidence, cannot determine the pros and cons of health outcomes

ATD: Antithyroid drug

B-blocker: Beta-blocker

$\mathrm{CH}$ : Congenital Hypothyroidism

DTC: Differentiated thyroid carcinoma

FT3: Free triiodothyronine

FT4: Free thyroxine

FNA: Fine needle aspiration

GTT: Gestational transient thyrotoxicosis

hCG: Human chorionic gonadotropin

LT4: Levothyroxine

MMI: Methimazole

NACB: National Academic of Clinical Biochemistry

PTU: Propylthiouracil

SCH: Subclinical Hypothyroidism

T3: Triiodothyronine

T4: Tetraiodothyronine

Tg: Thyroglobulin

TgAb: Thyroglobulin antibody

TPOAb: Thyroid perioxidase antibody 
TRAb: TSH receptor antibody

TSH: Thyroid stimulating hormone

TT4: Total thyroxine

UIC: Urinary iodine concentration

Copied from Guideline on diagnosis and management of thyroid disease during pregnancy and postpartum ( $2^{\text {nd }}$ edition) $\mathrm{Ad} \mathrm{Hoc}$ writing committee for guidelines on diagnosis and management of thyroid disease during pregnancy and postpartum; Chinese society of Endocrinology, Chinese Medical Association; Chinese Society of Perinatology; Chinese Medical Association.

This guideline is translated from original Chinese version to English version 
Table 5. Incidence and characteristics outcome of pregnancies managed for thyroid dysfunction.

\begin{tabular}{|c|c|c|c|}
\hline \multirow[t]{2}{*}{ Variables } & Marginal & Complication & \\
\hline & \multicolumn{3}{|l|}{$\mathbf{N}(\%)$} \\
\hline & & $\mathrm{OR}(95 \% \mathrm{Cl})$ & P-value \\
\hline Age & $724(100 \%)$ & $1.024(0.895-1.172)$ & 0.725 \\
\hline Parity & $724(100 \%)$ & $1.090(0.528-2.252)$ & 0.815 \\
\hline Gravida & $724(100 \%)$ & $1.368(1.028-1.821)$ & 0.032 \\
\hline Gestational age & $724(100 \%)$ & $1.100(1.073-1.127)$ & 0.000 \\
\hline \multicolumn{4}{|l|}{ Groups } \\
\hline G0 & $189(26.1 \%)$ & $1.005(0.398-2.533)$ & 0.992 \\
\hline G1 & $276(38.1 \%)$ & $0.938(0.401-2.195)$ & 0.882 \\
\hline $\mathrm{G} 2$ & $164(22.7 \%)$ & $2.009(0.693-5.823)$ & 0.199 \\
\hline G3 & $95(13.1 \%)$ & Reference & \\
\hline \multicolumn{4}{|l|}{ Thyroid dysfunction } \\
\hline Subclinical hypothyroidism & $420(58.0 \%)$ & $0.882(0.182-4.281)$ & 0.876 \\
\hline Hypothyroidism & $246(34.0 \%)$ & $0.798(0.163-3.912)$ & 0.781 \\
\hline Hyperthyroidism & $32(4.4 \%)$ & $2.411(0.274-21.197)$ & 0.427 \\
\hline Other thyroid dysfunction & $26(3.6 \%)$ & Reference & \\
\hline \multicolumn{4}{|l|}{ Method of delivery } \\
\hline Stillbirth/miscarriage & $7(1.0 \%)$ & - & - \\
\hline Normal vagina delivery & $341(47.1 \%)$ & $2.521(1.246-5.100)$ & 0.010 \\
\hline Assisted delivery & $26(3.6 \%)$ & $4.102(0.785-21.426)$ & 0.094 \\
\hline Caesarean section & $350(48.3 \%)$ & Reference & \\
\hline \multicolumn{4}{|l|}{ Age groups } \\
\hline $18-25$ & $98(13.5 \%)$ & $1.661(0.220-12.523)$ & 0.622 \\
\hline $26-34$ & $511(70.6 \%)$ & $1.629(0.513-5.177)$ & 0.408 \\
\hline$\geq 35$ & $115(15.9 \%)$ & Reference & \\
\hline
\end{tabular}


Table 6: Compared the relationship between groups and ages with various complications in Subclinical Hypothyroidism and Hypothyroidism.

\section{Complications}

Gestational HTN

GO

G1

$\mathrm{G} 2$

G3

Age

Preeclampsia/eclampsia

GO

G1

G2

G3

Age

Abortion(miscarriage)

GO

G1

G2

G3

Age

Preterm birth

\begin{tabular}{lll} 
G0 & 0.623 & 0.884 \\
\hline G1 & $0.978(1.022,0.218-4.780)$ & $0.792(0.795,0.144-4.374)$ \\
\hline G2 & $0.771(1.223,0.314-4.760)$ & $0.709(0.726,0.135-3.898)$ \\
\hline G3 & $0.353(0.423,0.069-2.601)$ & $0.445(0.453,0.059-3.459)$ \\
\hline Age & $0.429(1.045,0.937-1.164)$ & $0.926(1.006,0.893-1.132)$ \\
\hline Intrauterine fetal demise & & \\
\hline G0 & 1.000 & 0.366 \\
\hline G1 & $0.997(11028140.22)$ & $0.224(0.200,0.015-2.680)$ \\
\hline G2 & $1.000(1.175)$ & $0.106(0.131,0.011-1.539)$ \\
\hline G3 & $1.000(1.007)$ & $0.314(0.276,0.023-3.373)$ \\
\hline Age & $0.136(1.424,0.895-2.265)$ & $0.251(0.861,0.667-1.112)$ \\
\hline
\end{tabular}

\begin{tabular}{ll} 
Subclinical Hypothyroidism & Hypothyroidism \\
$\mathrm{N}=\mathbf{4 2 3}$ & $\mathrm{N}=\mathbf{2 4 7}$ \\
\hline P-value $(\mathrm{OR}, 95 \% \mathrm{Cl})$ & P-value(OR, 95\%Cl)
\end{tabular}

0.970

1.000

0.763(0.735, 0.099-5.433)

0.668(0.672, 0.110-4.123)

0.659(0.640, 0.088-4.660)

$0.390(1.066,0.922-1.232)$

0.838

0.883

$0.760(0.645,0.039-10.744)$

0.997

0.588(0.461, 0.028-7.603)

0.418(0.302, 0.017-5.478)

0.820(1.328, 0.115-15.280)

0.997

$0.059(1.196,0.993-1.441)$

$0.145(0.668,0.388-1.149)$

1.000

$1.000(0.897)$

0.998(16547540.01)

1.000(0.955)

$0.828(1.040,0.733-1.474)$

1.000(0.899)

(33524750.08)

$0.743(1.039,0.828-1.304)$

1.000

1.000(0.676)

0.997(33808903.68)

1.000(1.005)

0.018(1.459, 1.067-1.997)
0.998(32789142.07)

0.884 


\begin{tabular}{|c|c|c|}
\hline G0 & 0.261 & 0.222 \\
\hline G1 & $0.965(0.940,0.056-15.696)$ & $0.129(0.260,0.046-1.478)$ \\
\hline G2 & $0.249(3.480,0.417-29.003)$ & $0.048(0.152,0.024-0.981)$ \\
\hline G3 & $0.805(0.703,0.043-11.507)$ & $0.204(0.296,0.045-1.936)$ \\
\hline Age & $0.455(0.944,0.813-1.097)$ & $0.829(0.982,0.836-1.154)$ \\
\hline
\end{tabular}

\section{Figures}

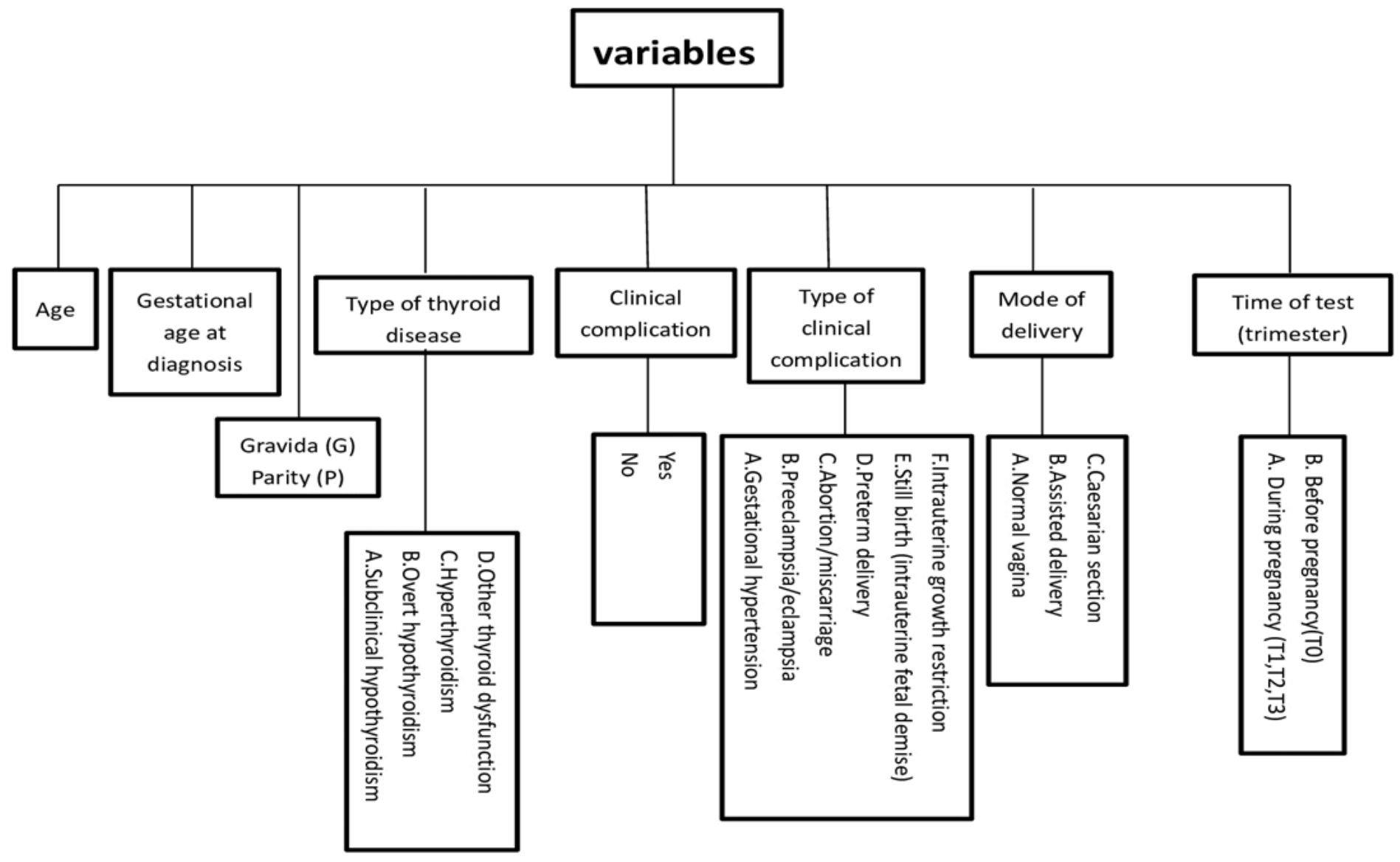

Figure 1

Parameters need for the study 


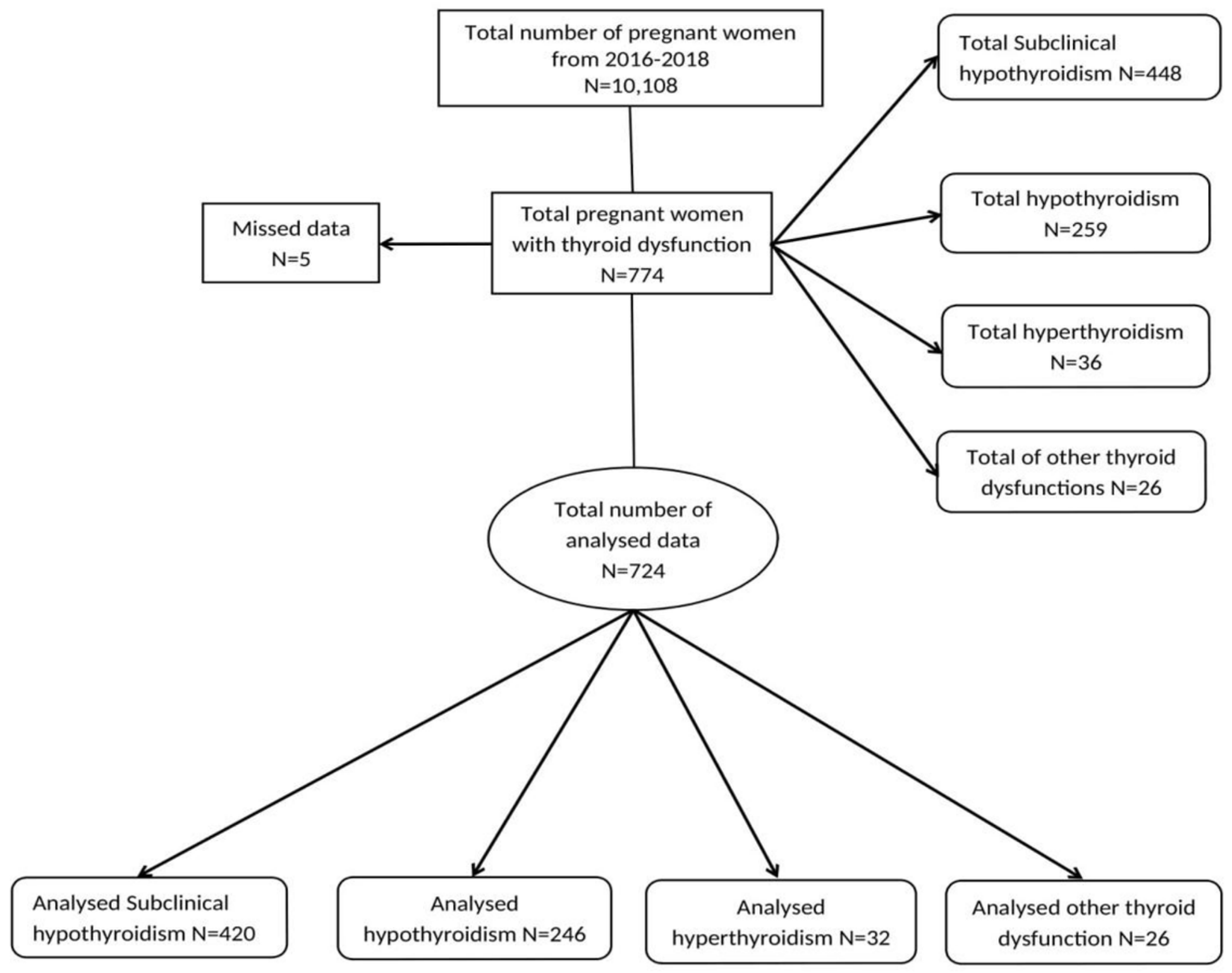

\section{Figure 2}

Outcome of patients who were screened and diagnosed with thyroid dysfunction and are included in the study. 